\title{
Advancement of Plasma Cold-Hearth Melting for Production of Gamma Titanium Aluminide Alloys within Arconic
}

\author{
Ernie Crist ${ }^{1}$, Birendra Jena ${ }^{1}$, Michael Jacques ${ }^{1}$, Matt Dahar ${ }^{1}$, Don $\operatorname{Li}^{1}$, Fusheng Sun ${ }^{1}$ \\ ${ }^{1}$ Arconic Engineered Structures, Niles, OH USA
}

\begin{abstract}
$\underline{\text { Abstract }}$
Utilization of gamma titanium aluminide alloys in aerospace and automotive/industrial applications has placed significant demand on melting sources for products to be used in cast, wrought, and direct-machining applications. There is also an increased demand for input stock used in gas atomization of powders. Current technologies used in ingot manufacturing include plasma arc melting, vacuum arc melting, and induction skull melting + centrifugal casting. Subsequent processing may include forging, remelting + casting, or machining directly into components. Over the past six years, Arconic Engineered Structures has developed a robust melting method using plasma cold-hearth melting technology, including the design and implementation of a new 3-torch system to produce Ti-48-2-2 cast bars. General discussions concerning plasma cold-hearth melting, manufacturing challenges, and metallurgical attributes associated with cast Ti-48-2-2 bars will be reviewed. Emphasis will be on understanding the impact of hot isostatic pressing on internal voids, residual stress cracking and resulting mechanical properties.
\end{abstract}

\section{$\underline{\text { Introduction }}$}

Next-generation aerospace propulsion systems are designed and manufactured to be more energy efficient. Utilization of gamma titanium aluminide low-pressure turbine blades represents one of many technologies that enable the GE LEAP and Pratt \& Whitney Geared Turbofan (GTF) engines to achieve their respective performance targets [1]. LEAP LPT blades utilize Ti-48Al$2 \mathrm{Nb}-2 \mathrm{Cr}$ machined from cast and heat-treated stock, while the GTF low-pressure turbine blades are produced from forged and heat-treated TNM alloy [2]. The large volume of titanium aluminide stock required to support the engine build rates is produced by a variety of methods, including VAR/ISM skull melting, and via plasma arc, cold-hearth melting (PAM) technologies. The complexity of gamma titanium aluminides presents many processing challenges, along with customer specifications impose very stringent requirements for qualifications and manufacturing procedures. The present paper describes the PAM technology and provides a review of two significant manufacturing challenges associated with using this melting method for production of Ti-482-2 titanium aluminide bars.

Plasma-arc, cold-hearth melting is defined as a semi-continuous process in which individual raw materials are compacted together and fed into a copper retort where they are melted, then progress through a refining hearth and poured into a water-cooled mold. The cast bar is lowered using a withdrawal mechanism while additional raw materials are added to the retort. The process continues until the desired cast bar length is achieved. An illustration of a 2-torch process is shown in Figure 1. A 3-torch system shown in Figure 2 incorporates a $\mathrm{C}$-shape hearth which provides improved refining capability due to increased residence time. Several ingot cross-sectional geometries can be cast, such as $150 \mathrm{~mm}$ round-cornered square (RCS), $125 \mathrm{~mm}$ diameter round, and $110 \mathrm{~mm}$ RCS with bar lengths of more than 3 meters.

Figure 3 provides a general representation of the thermal gradients and molten pool profile that exist during steady state melting and casting. Under normal process conditions, solidification occurs via nucleation and growth of columnar macro grains.

Examples are illustrated in Figures 4 and 5. Columnar grains grow radially toward the cast bar centerline and turn up slightly in the direction of heat input [3]. Melting parameters such as torch power and casting rate were developed and validated by Arconic at the production level in a way to provide consistent solidification behavior, while minimizing residual stresses and the propensity for internal cracking and segregation.
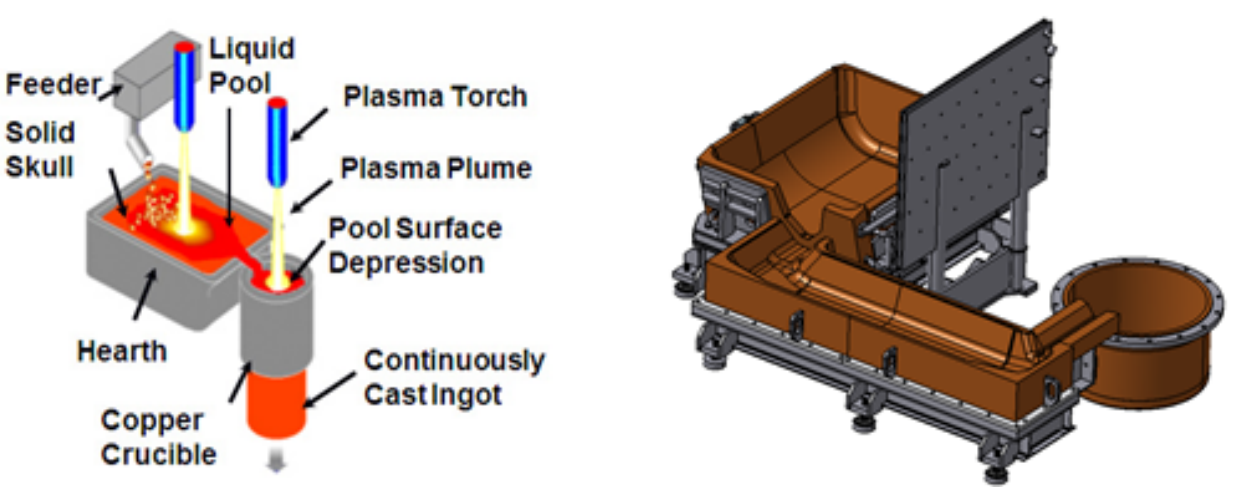


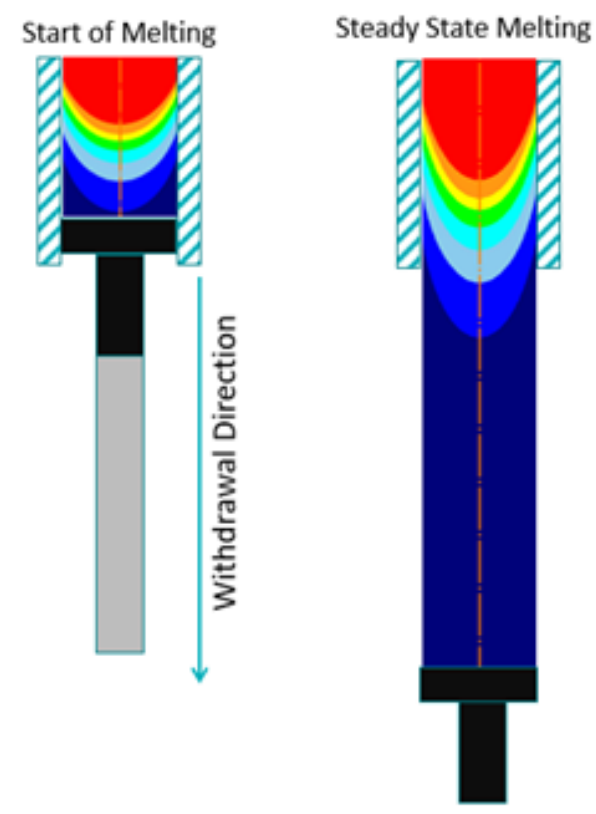

Figure 3. Generic representation of pool profile.

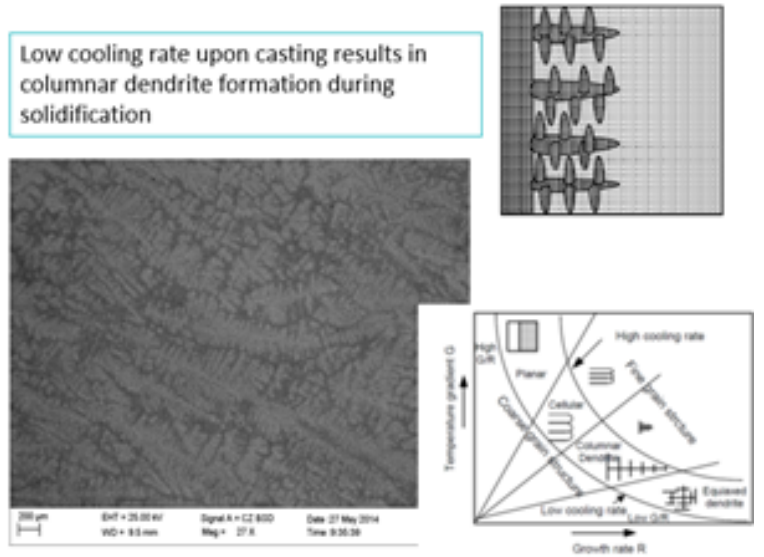

Figure 4. Example of columnar

dendritic growth [3].

As in all aspects of manufacturing, process interruptions will occur. In the case of plasma arc melting titanium aluminides, process interruptions can lead to delays in pouring, which can contribute to localized segregation. Variation in solidification due to bar cross-sectional area and casting rate may create residual stresses of enough magnitude to cause internal cracking. While hot isostatic pressing (HIP) is employed during the normal heat treat protocol, and it is assumed that all cracks not exposed to the surface will heal during HIP, it is not possible/practical to inspect for these internal cracks during normal production release testing. There were two primary objectives associated with this paper; (1) evaluate the process boundary conditions that result in acceptable microstructure and (2), simulate healing of an internal crack via HIP using a controlled experiment.
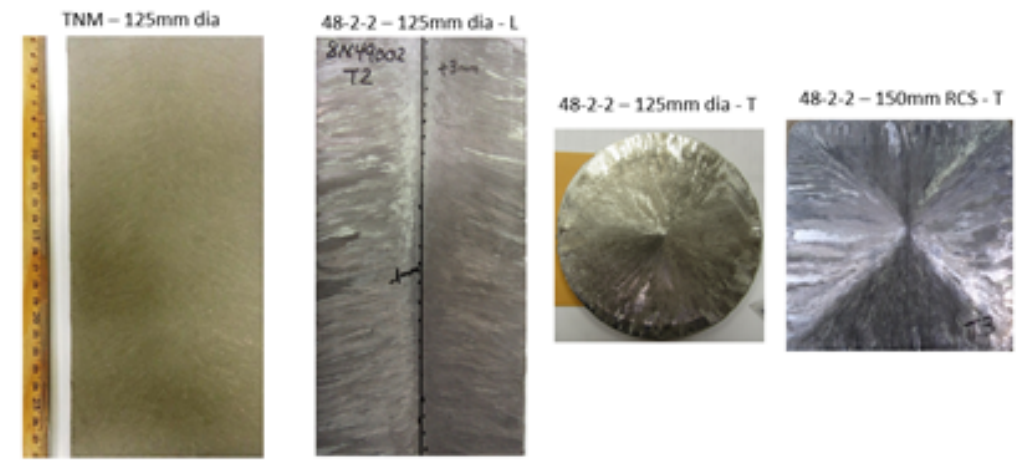

square mold configurations.

Figure 5. Representative macrostructure samples for TNM and 48-2-2 alloy in round and

\section{Experimental Procedure}

Microstructure uniformity is extremely sensitive to composition gradients, particularly aluminum variation [5]; consequently, understanding the influence of melting parameters on composition and hence, microstructure control is critical, particularly for material in rotating jet engine components. A controlled study using an Arconic plasma arc cold-hearth furnace was conducted to establish boundary conditions relative to melting interruptions and their associated impact on microstructure uniformity. 
Baseline parameters for the study consisted of those from initial process validation work performed by Arconic. Deliberate casting delays were introduced using multiples of the baseline parameters, ranging from $2 \mathrm{X}$ to $10 \mathrm{X}$. During the casting delays, the withdrawal mechanism was held in the stationary position, while the mold torch continued to dwell (at full power) on the liquid metal in the mold, without adding new material from the hearth. At the completion of each delay, normal melting (casting) conditions were returned to the system. Process data were used to correlate the physical location of the melt interruption in the cast bar so that test coupons could be excised from those exact locations.

The cast bar containing the deliberate melting interruptions was subjected to the production thermal cycles consisting of HIP and post-HIP heat treatment. Longitudinal macrostructure slices excised from the centerline and corresponding microstructure specimens were excised from the locations representing the melting interruptions. Optical and scanning electron microscopy were utilized to correlate microstructure features with their respective casting delay parameters.

To simulate healing of an internal crack by diffusion bonding, a novel fabrication approach was taken. Cylindrical sections of as-plasma melted Ti-48-2-2 titanium aluminide bars ( 100mm diameter x $125 \mathrm{~mm}$ long) were completely machined (OD and each end face) to a surface roughness of 32Ra. The goal was to produce mating surfaces that were parallel to each other, while minimizing surface asperities. Machined dimensions were selected based on the ID and length of CP Grade 2 titanium pipe. One end of the pipe was sealed via electron beam welding a machined end-cap. The two titanium aluminide segments were placed inside of the pipe, and the machined ends were in direct contact with each other. The fabrication was placed inside of the EB welding chamber which utilized a vacuum level of $9 \times 10^{-4}$ Torr. The second end cap was secured in place using the EB welding process, ensuring the titanium aluminide segments were sealed under an extremely high vacuum level. The assembly was fabricated in a way that prevented any internal movement of the titanium aluminide segments within the pipe. An illustration of the assembly is shown in Figure 6. Cam et al demonstrated that successful diffusion bonding of titanium aluminides was performed at $925-1150^{\circ} \mathrm{C}$ at an internal operating pressure of $4.5 \times 10^{-5}$ Torr utilizing conventional SPF/DB technology [4]. In their experiments, diffusion bonding was performed in the range using 20-40MPa of external pressure [4]. In the current scope of work, the titanium aluminide samples were under a vacuum level comparable to Camâ€ $€^{\mathbf{T M}_{S}}$ work, but the bonding (HIP) temperature was slightly higher, while pressure and time were nearly $4 \mathrm{X}$ of that used by Cam [4].

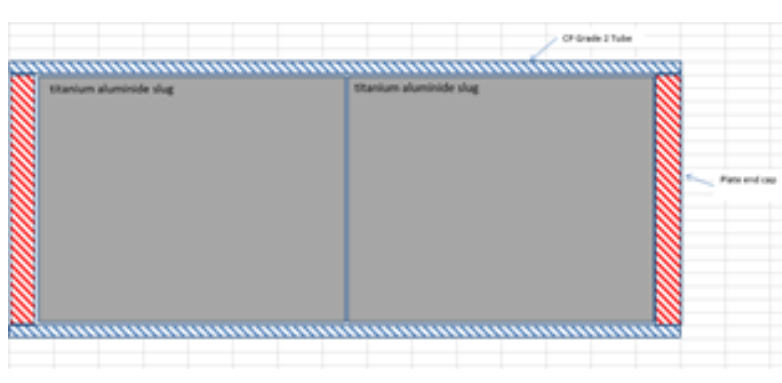

Figure 6. Illustration of fabrication details.

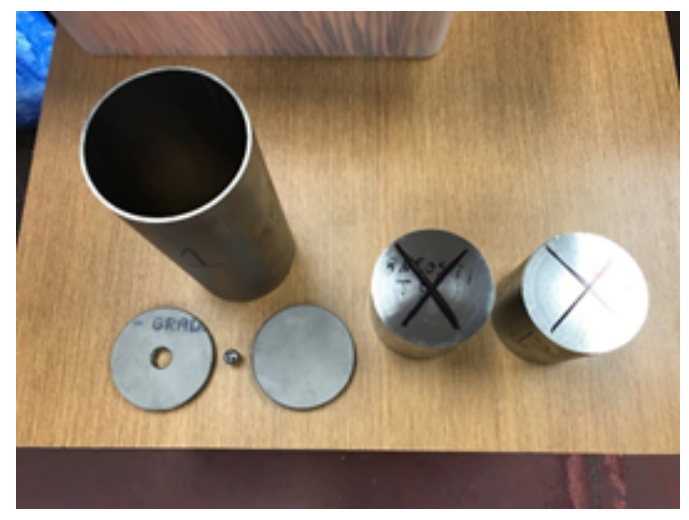

Figure 7. Components for welded assembly.

The welded assembly was subjected to a post-weld thermal cycle that included hot isostatic pressing for 4 hours at $1185^{\circ} \mathrm{C}$ and $144 \mathrm{MPa}$ pressure, followed by 7 hours at THIP. HIP temperature was comparable to that used by G. Cam et al, but pressure was substantially higher [4]. Fabricated components were heat treated with normal production material. Upon completion of the thermal cycles, samples for microstructure analysis and tensile testing were excised in such a way as to ensure the bond line was at the center of the test coupons. The purpose of the metallurgical assessment was to validate diffusion bonding of the TiAl-TiAl interface, and to understand the microstructure transformation at the original crack interface.

\section{$\underline{\text { Results }}$}

\section{Casting Interruption Studies}

Longitudinal macrostructure sections from the casting interruption trials are shown in Figure 8 . These photographs represent the result of casting delays ranging from the baseline timeframe up to $4 \mathrm{X}$ baseline. The more pronounced effect can be seen near the outer edge of the cast bar. Outlines of prior molten pool is observed by the parabolic shape visible within the macro slice. It is more evident on the zone representing $4 \mathrm{X}$ baseline. The micrographs in Figure 9a-9d provide a higher magnification view across the solidification front associated with the casting interruption designated $4 \mathrm{X}$ baseline. The photographs indicate the transition from $100 \%$ lamellar (9a) to the more typical gamma + lamellar, as in 9d, moving counter-clockwise from 9a. 


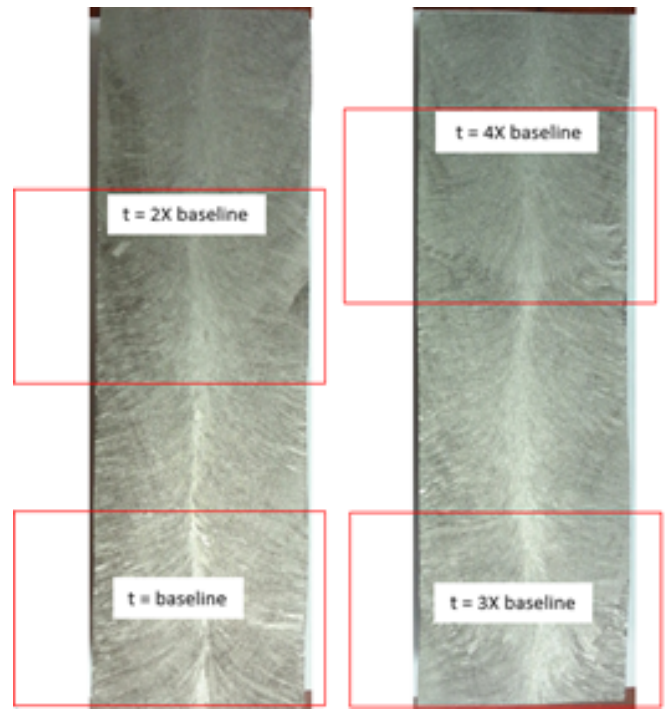

Figure 8. Longitudinal macrostructures

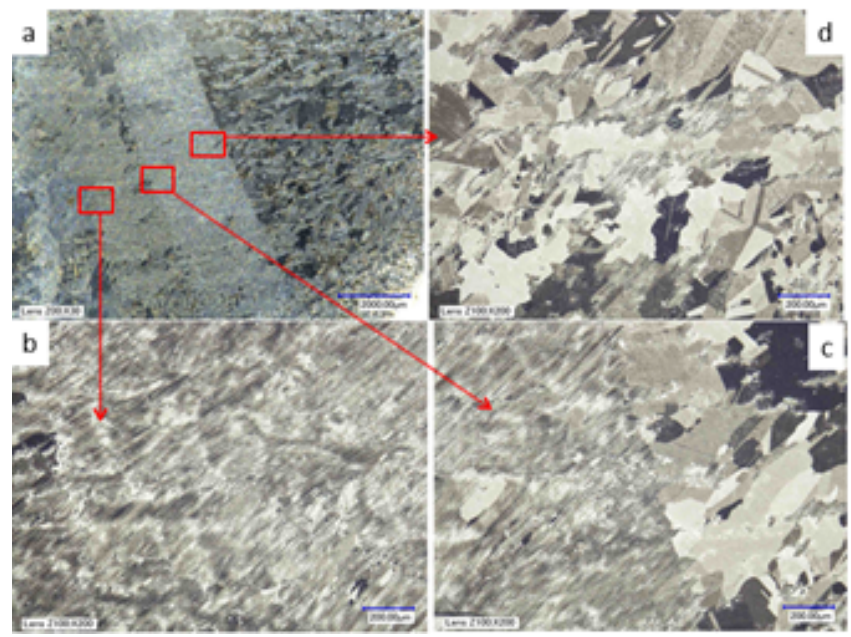

Figure 9. Microstructure

gradients across solidification front

Figure 10 designates the physical locations across the transition zone for $4 \mathrm{X}$ baseline at which SEMEDXA chemistry analyses were performed. The left edge of the photographs represents the outer edge of the cast bar. The transition line indicates the approximate position in the melting when casting withdrawal stopped, while the mold torch continued to dwell on the molten pool established during that period. The semi-quantitative data shown in Table I represent the composition gradient across the transition zone noted in Figure 9a and Figure10. The lower aluminum content most likely explains the $100 \%$ lamellar content in the SEM-EDXA locations denoted as 1-5 inclusive.

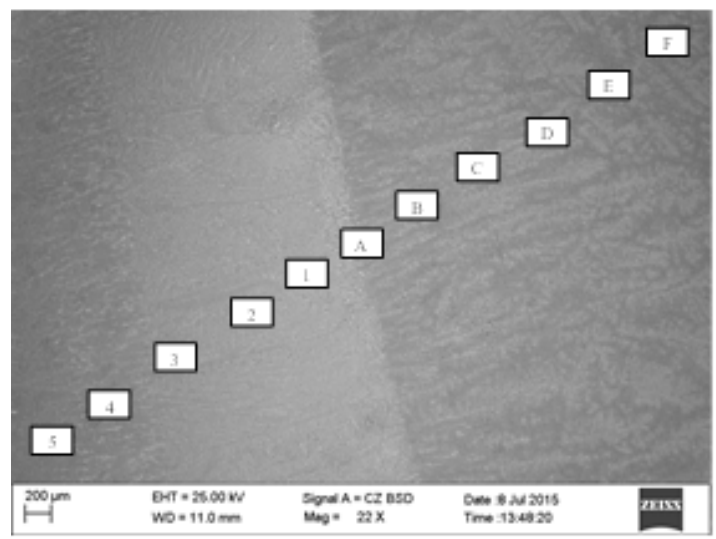

Figure 10. Location of SEM-EDXA analyses

Table I. SEM-EDXA analysis across the transition zone for the $4 \mathrm{X}$ baseline location. 


\begin{tabular}{|c|cc|ccc|c|c|cccc}
\hline IDs & $\mathbf{5}$ & $\mathbf{4}$ & $\mathbf{3}$ & $\mathbf{2}$ & $\mathbf{l}$ & $\mathbf{A}$ & $\mathbf{B}$ & $\mathbf{C}$ & $\mathbf{D}$ & $\mathbf{E}$ & $\mathbf{F}$ \\
\hline Al & 29.34 & 29.46 & 29.81 & 30.00 & 29.75 & 29.84 & 30.37 & 30.54 & 30.55 & 30.45 & 30.46 \\
& & & & & & & & & & & \\
\hline Nb & 04.57 & 04.72 & 04.43 & 04.55 & 04.68 & 04.69 & 04.56 & 04.68 & 04.59 & 04.70 & 04.73 \\
Ti & 63.34 & 63.14 & 63.17 & 62.95 & 63.14 & 62.95 & 62.50 & 62.39 & 62.37 & 62.35 & 62.36 \\
\hline Cr & 02.75 & 02.69 & 02.60 & 02.49 & 02.42 & 02.52 & 02.57 & 02.39 & 02.49 & 02.51 & 02.45 \\
\hline
\end{tabular}

\section{Diffusion Bonding Studies}

The second phase of the present work involved a methodology intended to simulate healing or diffusion bonding of internal cracking during HIP. Under the bonding conditions studied by Cam et al, equiaxed gamma grains formed at the original bond line due to dynamic recrystallization resulting from deformation of surface irregularities during the application of the diffusion bonding pressure [4]. The illustration in Figure 11 shows the vertical mating plane in the center of the left image, nucleation of new gamma grains along the interface, center image followed by growth of the new grains during post-bonding thermal treatments [4].
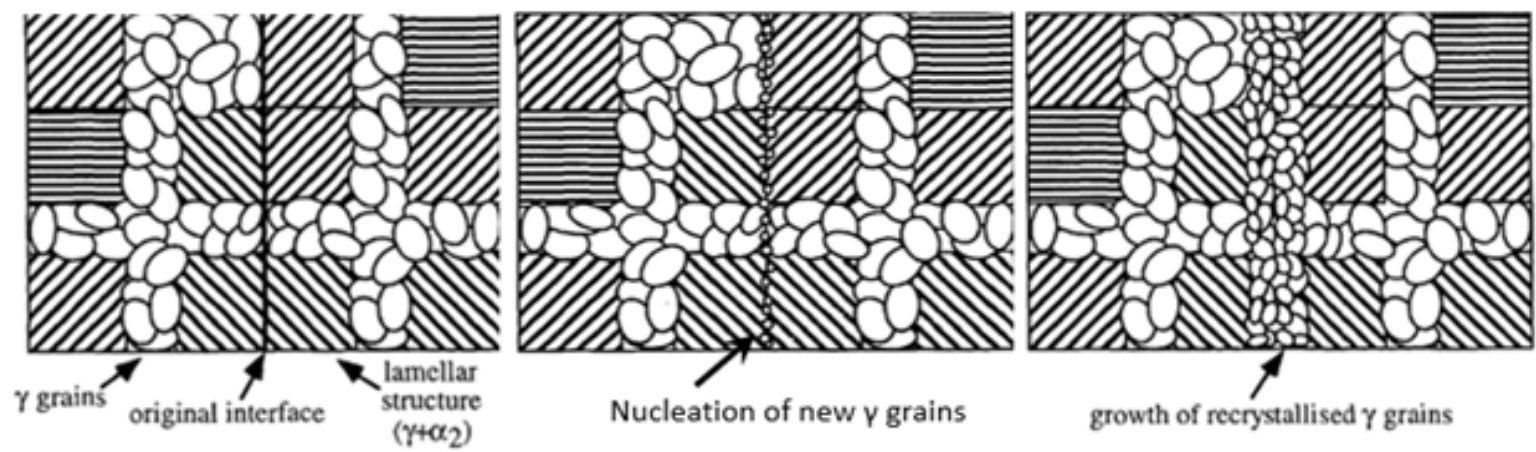

Figure 11. Illustration of grain nucleation and growth at bond interface [4]

A longitudinal cross-section of the bonded assembly is shown in Figure 12. The box highlights the physical location of the bond line. Optical micrographs for the present work are shown in Figures 13 and 14. The typical photomicrograph of bonded area away from the bond interface is shown in Figure 13. The microstructure consists predominantly of gamma phase, representative of the parent material used for diffusion bonding. The microstructure at the diffusion bonding interface in Figure 14 shows a seamless transition with nucleation of small gamma particles at the bond line. The features are comparable to those in Figure 11 illustrations. The white phase at bond interface seen in the back scattered electron micrograph of Figure 15 appears to be beta phase as the EDS microanalysis showed presence of higher chromium content. Evidence of beta phase was also present in microstructure locations several centimeters away from the bond interface.
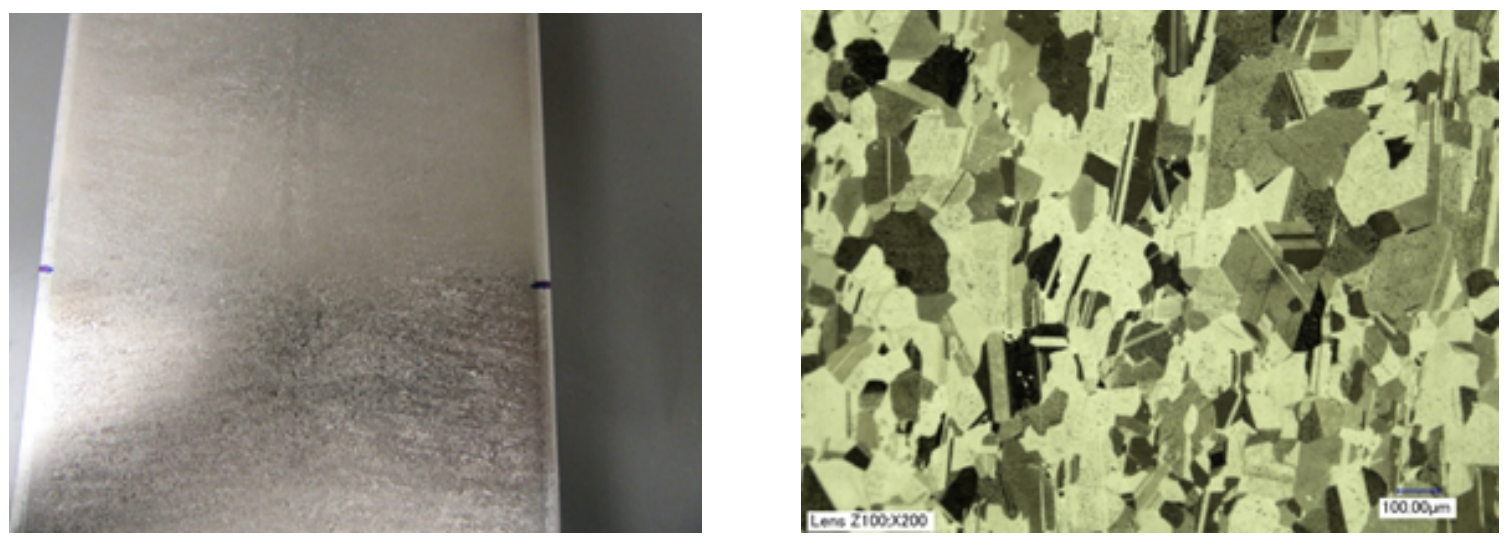

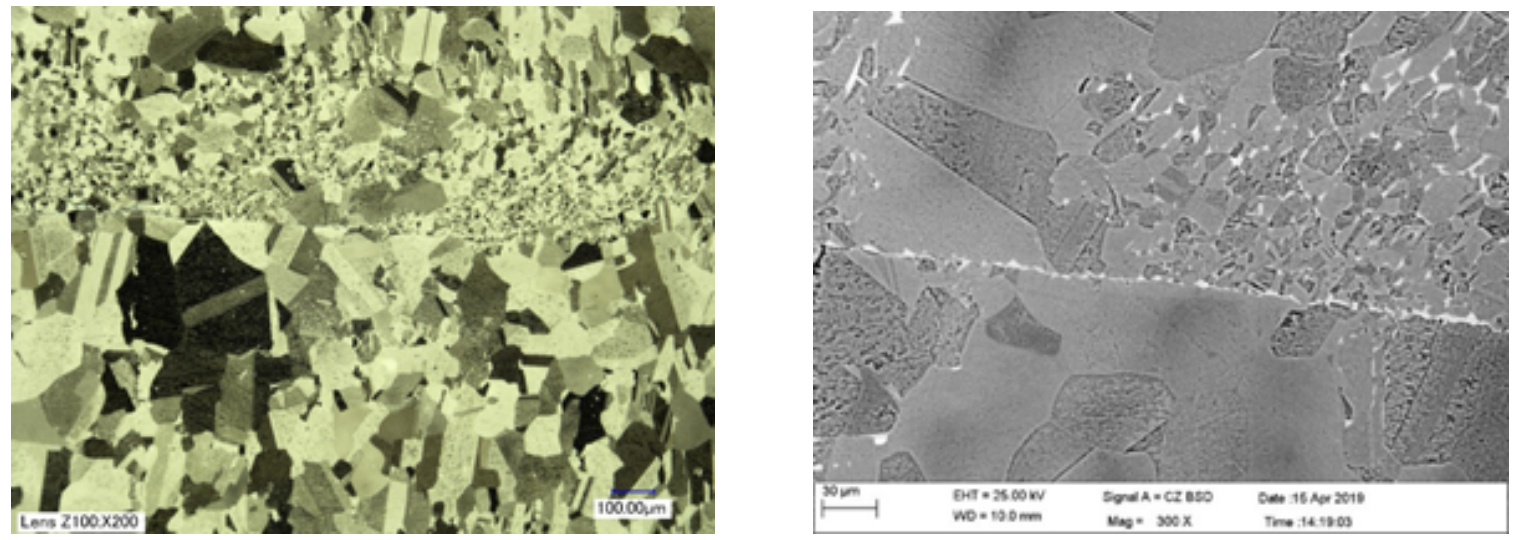

image at the bond interface

Figure 14. Bond line microstructure (horizontal)

Figure 15. BSE

Room temperature tensile tests were performed, whereby the bond interface was in the center of the tensile bar gage length. Machining and testing were conducted in accordance with the same protocol used for normal production testing. Data in Figure 16 represents yield strength data for production material. An interval plot shows a 95\% confidence level for the mean of each group. The results for the diffusion bonded specimens are within the dataset for melt process 2 . It is noted here that melt process refers to the physical geometry of the cast bar manufactured in the PAM furnace. The current study utilized material from melt process 2 . Yield strength results were within the normal scatter band for this process. Room-temperature elongation values for this material are typically $<0.5 \%$ [6]. Results from the bond interface testing were also within the normal scatter exhibited by production material.

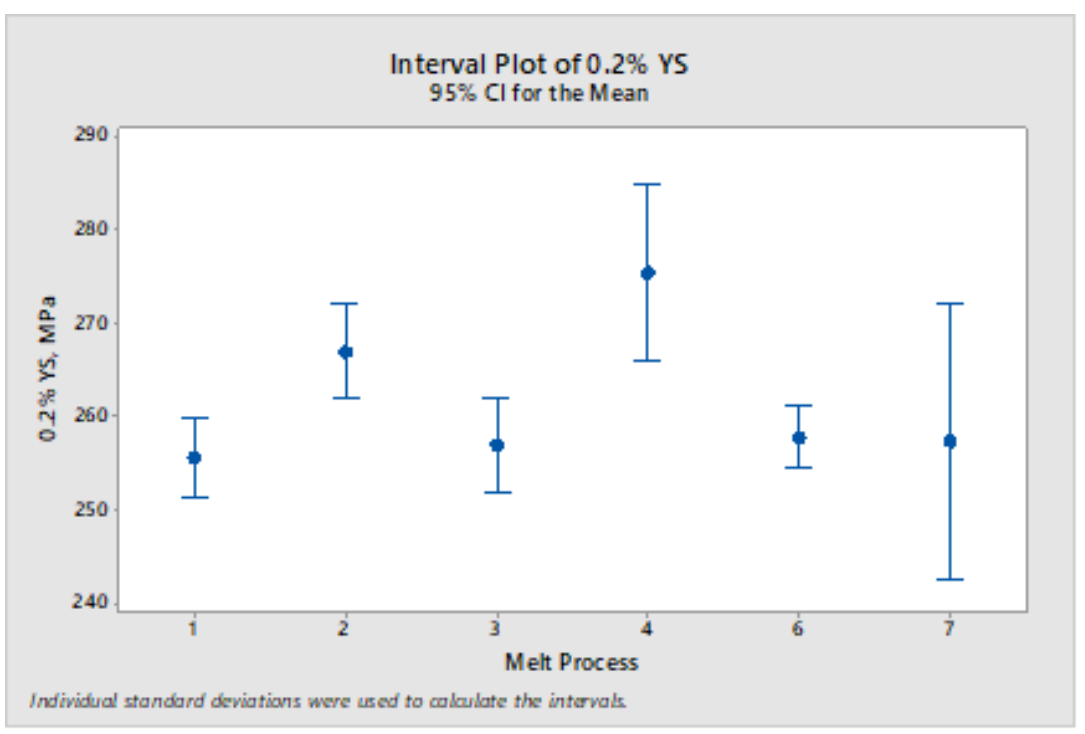

Figure 16. Yield strength data including bond interface

\section{$\underline{\text { Discussion }}$}


The casting delays noted in Figures 8-10 resulted in some aluminum evaporation, leading to the macro/micro/composition gradients noted in the longitudinal sections. The decreased aluminum content contributed to the large percentage of lamellar microstructure, particularly near the outer edge of the cast bar. At the mid-radius and center locations, there were no discernable microstructure differences across the boundary denoted by the casting delays [5]. These analyses provided useful information regarding establishment of process boundary conditions, whereby process parameters were correlated with macro and microstructure results and compared to limits of acceptability imposed by Arconicâ€ ${ }^{\mathrm{TM}_{\mathrm{S}}}$ customers.

The second segment of the present work focused on a methodology to simulate healing of internal cracks that originate during casting and solidification. While it is possible to machine and perform non-destructive testing on the bars produced in the PAM furnace, that is approach is not practical on a production-scale. Section sizes are too large for x-ray inspection, and grain noise/attenuation make ultrasonic inspection not feasible. The demonstration completed in this scope of work provided evidence that it was possible to diffusion bond a rather large interface $\left(>78 \mathrm{~cm}^{2}\right)$ that is expected to be significantly larger than any residual crack present in production material. HIP pressure and temperature were both higher than other titanium aluminide diffusion bonding work found in the public domain. Microstructure and tensile test results across the bond interface were found to be comparable to those in the published work [4]. The data provides a level of confidence in the ability to heal internal cracks that can result from residual stress during solidification and cooling of the cast bars.

\section{Conclusions}

Results of the present casting interruption work provided objective evidence used for the development of fixed manufacturing routes and process boundaries. It allowed definition of permissible process parameter variation and the impact on product attributes such as macrostructure and microstructure behavior. These data points have since been successfully validated through extensive analyses of production bars over an extended period.

Defect-free bond was achieved using the assembly prepared using electron-beam melting chamber under a high vacuum level, and through the prescribed HIP and post-HIP heat treatment. Physical and mechanical testing of the diffusion-bonded interface indicated that the interface behaved like base material. Although the interface showed the presence of finer gamma grains, they did not adversely affect the material. Results from this study were comparable to those achieved by Cam et al and indicate that any internal cracking that results from residual stress will adequately heal utilizing the customer-prescribed HIP parameters. Destructive analyses performed on numerous titanium aluminide bars (conducted outside of the present study) did not reveal the presence of any internal cracking. Those results, in conjunction with the results from the present study provide confidence that any internal cracks generated during or post-solidification healed during HIP.

\section{Acknowledgements}

The authors would like to thank the Arconic Engineered Structures Research and Development technicians for their efforts relative to execution of the manufacturing trials, and significant work preparing samples for macrostructure and microstructure analysis. Special thanks go to Mr. Daniel Posniack (student) at Ohio State University for facilitating the machining of specimens, electron beam welding and heat treatment work related to the diffusion bonding study. EB Tech in Agawam, MA is responsible for the excellent electron beam welding that contributed to the success of the diffusion bonding experiment.

\section{$\underline{\text { References }}$}

[1] B. P. Bewlay, M. Weimer, T. Kelly, A. Suzuki, and P.R. Subramanian, â€œIntermetallic-Based Alloy â€“ Science, Technology and Applications,â€w in Intermetallic-Based Alloy â€“ Science, Technology and Applications, Pittsburgh, PA, USA, 2013, vol. 1516, pp. 49-58.

[2] D. Hautmann, Report, MTU Aero Engines 1/2013, pp. 24-29.

[3] T. Udemphol, The Weld Microstructure, Suranaree University of Technology, September 2007

[4] G. Cam, J. Mullauer, M. Kocak, â€œDiffusion Bonding of Two-Phase Gamma TiAl Alloys with Duplex Microstructure,â€w Science and Technology of Welding and Joining (1997) 213-219.

[5] F. Sun, E. Crist, Alcoa Titanium \& Engineered Products internal report, 2017.

[6[ B. Jena, E. Crist, Arconic Engineered Structures internal report, 2018. 